\title{
AÇÃO DE DIVERSOS INSETICIDAS SOBRE Stiphra robusta MELLO LEITÃO, 1939 (Orthoptera, Proscopiidae) EM Eucalyptus citriodora
}
F.N.P. HAJI ${ }^{1}$
S.M. SOUZA ${ }^{1}$
$\mathrm{J} . \mathrm{C} \cdot \mathrm{TOSCANO}^{2}$

\author{
ABSTRACT
}

Effect of different insecticides on Stiphra robusta Mello Leitão, 1939 (Orthoptera, Proscopiidae) in Eucalyptus citriodora

The present work was conducted to evaluate the efficiency of different insecticides for the control of Stiphra robusta Mello Leitão, 1939, a severe pest of Eucalyptus citriodora, in Petrolina, PE, Brazil. The experiment was conducted in a randomized complete-block design with seven treatments, four replications, and four plants/plot. The treatments consisted on the evaluation of the following insecticides and respective dosages: a. control (no insecticide); b. endosulfan $35 \mathrm{EC} 2.5$ l/ha; c. triazofos $40 \mathrm{BR}, 15 \mathrm{l} /$ /ha; d. endosulfan + methyl parathion. $2.0 \mathrm{l} / \mathrm{ha} ; \mathrm{e}$. deltamethrin $2.5 \mathrm{EC}, 0,4 \mathrm{l} / \mathrm{ha}$; f. fenvalerate $30 \mathrm{EC}, 0.3 \mathrm{l} / \mathrm{ha}$, and methyl parathion $60 \mathrm{Em}, 1.5 \mathrm{l} / \mathrm{ha}$. The percentage of efficency was obtained by ABBOTT's (1925) formula, from the percentage of infestation. The most efficient treatments were fenvalerate $30 \mathrm{EC}(83.72 \%$ of control) and deltamethrin $2.5 \mathrm{EC}$ (79.42\% of control) which differred statistically from the others, but not between themselves. The num ber of insects per plant was obtained at 5, 10, 24, 48, and $12 \overline{0}$ hours after the application of the insecticides, using a $2 \times 2$ black plastic sheet beneath the plants.

Recebido em 28/03/84

1 Embrapa/CPAtSA, Caixa Postal 23, 56300 - Petrolina, Pe.

2

HOESCHT DO BRASIL. 


\section{INTRODUCAAO}

Stiphra robusta Mello Leitão, 1939 ocorre segundo MELLO LEITÃO (1939) e SILVA et alii (1968), no Nordeste brasileiro, nos Estados de Pernambuco, Ceará, Paraíba e Rio Grande do Norte, encontrando-se atualmente disseminado na Bahia e Piauí, conforme MoRAES et $a$ li (1980).

Essa praga, vulgarmente conhecida por "mané magro", "gafanhoto da jurema", "Maria seca" ou "Maria mole", causa severos danos em cajueiro (CAVALCANTE et a $i i i$, 1975), frutiferas (BASTOS \& ALVES, 1979), maniçoba (BASTOS et a $i$ i, 1979), eucalipto (MORAES alii, 1981) e plantas da caatinga de importáncia madeireira, produ zindo uma total desfolha.

Observa-se que a medida que as plantas da caatinga perdem a folhagem, o "mané magro" passa a atacar plantas cultivadas como algodão, sorgo, leucena, mandioca, jojoba, eucalipto e outras.

A população desse inseto nos últimos anos, tem atingido niveis bastante elevados e apresentando uma maior aiversificação de hospedeiros. Entretanto, nem todas as espécies de plantas são igualmente atacadas. BASTOS (1975b) no Ceará, em estudos de preferência de $S$. robusta por algumas plantas cultivadas, verificou que - cajueiro e a mangueira foram as preferidas. MORAES et alii (1981), estudaram o comportamento de várias espécies de Etealyptıs ao ataque de S. robusta e verificaram que E. camaldulensis, E. ereabra, $E$. nesophiza e E. polucarpa foram menos danificadas.

De 1979 a 1982, período de baixa precipitação pluviométrica, observou-se um considerável aumento da população de s. robusta. No Arizona, NERNEY \& HAMILTON (1969), verificaram que no inicio da primavera, a precipitação elevada, propicia o crescimento preco ce das espécies hospedeiras de gafanhoto, favorecendo o desenvolvi mento de sua população. MORAES et alii (1980), atribuiram o grandē surto de $S$. robusta à baixa pluviosidade, registrada de junho de 1978 a março de 1979. Fato idêntico foi também observado por coSENZA (1977) em Minas Gerais, porém, com espécies de acridideos.

Considerando-se a severidade dos danos causados por S. robusta em essências florestais e a sua elevada infestação, realizou-se este trabalho, cujo objetivo foi avaliar a eficiência de diver sos inseticidas no controle desse proscopideo, em E. citriodorá, por ser esta espécie uma das mais atacadas.

\section{MATERIAL E METODOS}

Este trabalho foi conduzido na área experimental de Manejo da Caatinga do Centro de Pesquisa Agropecuária do Trópico Semi-Ārido pertencente a Empresa Brasileira de Pesquisa Agropecuária (CPATSA-EMBRAPA), em Petrolina - PE e instalado em 23 de junho de 1982. 
As plantas de $E$. citriodora apresentavam em média $3,5 \mathrm{~m}$ de al tura, com um ano de idade, numa área de 1 ha tendo um espaçamentō de $3 \times 2 \mathrm{~m}$.

o delineamento experimental utilizado foi de blocos ao acaso, com quatro repetições e com quatro plantas por parcela, dos seguin tes tratamentos: 1) endosulfan $35 \mathrm{CE} 2,5 \mathrm{l} / \mathrm{ha} ; 2$ ) triazofos $40 \mathrm{~B} \overline{\mathrm{R}}$ $1,51 /$ ha; 3) endosulfan + paration metil 2,0 1/ha; 4) deltametrina 2,5 CE 0,4 1/ha; 5) fenvalerato $30 \mathrm{CE} \mathrm{0,3} \mathrm{1/ha;} \mathrm{6)} \mathrm{paration} \mathrm{metil}$ $60 \mathrm{Em} .1,5 \mathrm{l} / \mathrm{ha} ;$ 7) testemunha.

o equipamento de aplicação empregado foi um atomizador costal motorizado.

A contagem do número de insetos mortos por planta foi realiza da com 5, 10, 24, 48 e 120 horas apos aplicação, utilizando-se sō as plantas, um lençol plástico, preto, medindo 2 x $2 \mathrm{~m}$. Após a última contagem, fêz-se uma aplicação de choque com deltametrina, na dosagem de 2,0 l/ha. Avaliou-se a eficiência dos produtos pela fór mula de ABBOTT, (1925) através da percentagem de infestação dośs tratamentos e, a média dos dados foram transformados em arc. sen $\sqrt{\circ}$.

\section{RESULTADOS E DISCUSSÃO}

No Quadro 1, mostram-se os dados referentes aos tratamentos, percentagem de mortalidade de $S$. robusta, percentagem de eficiència dos tratamentos e contraste da média dos dados transformados em arc. sen $\sqrt{\circ}$, avaliados pelo teste de Tukey. Por estes resultados, verificou-se por ordem decrescente de eficiência que os melho res inseticidas foram: fenvalerato $(83,70 \%)$ e deltametrina $(79,42 \%)$, os quais diferiram estatisticamente dos demais mas não diferiramen tre si; paratiom metil $(54,22 \%)$, triazofós $(48,67 \%)$, endosulfan + + paratiom metil $(21,58 \%)$, e endosulfan $(11,67 \%)$, o qual estatisti camente não diferiu da testemunha. Para o paratiom metil e triazofós, estes resultados não concordam com os obtidos por BASTOS (1975a) e BASTOS \& ALVES (1979), em ensaios em campo e laboratório, respectivamente, onde esses produtos foram os mais eficientes. Para o endosulfan os resultados foram idênticos.

CONCLUSAOO

Com base nos resultados obtidos neste trabalho, que fenvalerato e deltametrina foram os produtos mais no controle de $S$. robusta. verifica-se eficientes 
QUADRO 1 - Tratamentos, dosagem, percentagem de mortalidade de Stiphra robusta Mel1o Leităo, 1939, percentagem de eficiência calculada pela fórmula de ABBOTT e média dos dados transformados em arc. sen $\sqrt{\%}$. Petro lina - PE, 1982.

\begin{tabular}{|c|c|c|c|c|c|c|c|}
\hline \multirow[b]{2}{*}{ Tratamentos } & \multirow[b]{2}{*}{$\begin{array}{l}\text { Dosagem } \\
(1 / \mathrm{ha})\end{array}$} & \multicolumn{4}{|c|}{ Percentagem Mortalidade } & \multirow{2}{*}{$\begin{array}{l}\text { Percenta- } \\
\text { gem efici } \\
\text { ência }\end{array}$} & \multirow{2}{*}{$\begin{array}{l}\text { Média dos da- } \\
\text { dos transfor } \\
\text { mados em arc. } \\
\text { sen } \sqrt{\%}^{\text {a }}\end{array}$} \\
\hline & & I & I I & I I I & IV & & \\
\hline Endosulfan & 2,5 & 15,79 & 9,37 & 30,76 & 18,07 & 11,67 & $25,04 \mathrm{~cd}$ \\
\hline Triazofós & 1,5 & 47,00 & 57,77 & 58,33 & 45,90 & 48,67 & 46,24 b \\
\hline \multicolumn{8}{|l|}{ Endosulfan + } \\
\hline Paratiom metil & 2,0 & 21,56 & 26,92 & 24,56 & 41,81 & 21,58 & $32,24 \quad c$ \\
\hline Deltametrina & 0,4 & 74,73 & 76,25 & 78,51 & 87,17 & 79,42 & $63,15 a$ \\
\hline Fenvalerato & 0,3 & 83,45 & 82,96 & 79,03 & 87,69 & 83,70 & $66,34 a$ \\
\hline Paration metil & 1,5 & 50,86 & 58,58 & 59,57 & 61,11 & 54,22 & $49,21 b$ \\
\hline Testemunha & - & 2,89 & 13,79 & 12,85 & 2,32 & 6,53 & 15,31 \\
\hline
\end{tabular}

$$
\text { DMS }=10,79 \quad \mathrm{CV}=11,01 \%
$$

a Médias seguidas da mesma letra, não diferem estatisticamente entre si, pelo teste de Tukey ao nivel de $5 \%$. 


\section{LITERATURA CITADA}

ABBOTT, W.S. A method of computing the effectiveness of an insecti cide. J. econ. Ent. 18: 265-267, 1925.

BASTOS, J.A.M. Ensaio de controle do "Mané magro", Stiphra robusta M-T, com inseticidas orgânicos sintéticos, em campo. Eitossanidade, Fortaleza, 1(2): 54-55,1975a.

BASTOS, J.A.M. Estudo preliminar de preferência do "Mané magro" Stiphra robusta M-T, por algumas plantas culltivadas. Fitossanidade, Fortaleza, 1(3): 90-91, 1975b.

BASTOS, J.A.M. \& ALVES, V.P.O. Ensaio de controle do "Mané magro", Stiphra robusta Lt., com inseticidas orgânicos sintéticos em laboratório. Fitossanidade, Fortaleza, 3(1/2): 20-21, 1979.

BASTQS, J.A.M.; FLECHTMAN, C.H.W.; FIGUEIREDO, R.W. de. Subsidios para o conhecimento das pragas da maniçoba. Fitossaniadale, Fortaleza, 3(1/2): 45-46, 1979 .

CAVAlCANTE, R.D.; CAVAiCANTE, M.L.S.; SANTOS, O.M. de L. Stiphra robusta Leitão, 1939, atacando cajueiro no Ceará. Fitossanidade, Fortaleza, 1(3): $94,1975$.

CONSENZA, G.M. Uso da aplicação aérea e terrestre de inseticidaspa ra o controle do gafanhoto em Minas Gerais. An. Soc. Entomol. Brasiz, 6(2): 295-300, 1977 .

MELLO-LEITÃo, C. de. Estudio monografico de los Proscopidos. Revta Mus. La Plata, Secc. Zool. 1(8): 279-445, 1939.

MORAES, G.J. de; LIMA, P.C.F.; SOUZA, S.M. de; SILVA, C.M.M. de S. Surto de Stiphra bitaeniata, Leitão (Orthoptera: Proscopiidae) no Trópico Semi-Árido. Ecosistema, 5:96-99; 1980.

MORAES, G.J. de; PIRES, I.E.; SOUZA, S.M. de; RIBASKI, J.; OLIVEIRA, C.A. de V. Resistência de espécies de Eucalipto ao ataque de Stiphra robusta, Mello Leitão (Orthoptera: Proscopiidae). B. téc. SIF, Viçosa, MG, (10): 1-7, 1981.

NERNEY, N.J. \& HAMILTON, A.G. Effects of rainfall on range forage and populations of grasshoppers, San Carlos Apache Indian Reser vation, Arizona, J. econ. Ent. 62(2): 239-243, 1969.

SILVA, A.G. A'A.; GONÇALVES, C.R.;GALVÃO, D.M.; GONÇALVES, A.J.L.; GOMES, J.; SILVA, M. do N.; SIMONI, L. de. Quarto catálogo dos insetos que vivem nas plantas do Brasil: seus parasitas e preda dores; Rio de Janeiro, Ministério da Agricultura, Departamento de Defesa e Inspeção Agropecuária, Laboratório Central de Patologia Vegetal, 1968. T.1, parte 2, 622p e T.2, parte 2, 265p. 


\section{RESUMO}

o presente trabalho teve como finalidade, avaliar a eficiência de diversos inseticidas no controle de Siiphra robusta Mello Leitão, 1939, séria praga de Écalyptus citriodora, em Petrolina - PE. O delineamento experimental empregado foi o de blocos ao aca so, com sete tratamentos e quatro repetições com quatro plantas por parcela. Avaliaram-se os inseticidas endosulfan 35 CE 2,5 1/ha, triazofós 40 BR 1,5 l/ha, endosulfan + paratiom metil 2,0 1/ha, del tametrina $2,5 \mathrm{CE} 0,4 \mathrm{l} / \mathrm{ha}$, fenvalerato $30 \mathrm{CE} 0,31 / \mathrm{ha}$, paratiom metil $60 \mathrm{Em} .1,5 \mathrm{l} / \mathrm{ha}$ e a testemunha. A percentagem de eficiència foi obtida pela fórmula de ABBOTT (1925) através da percentagem de infestação dos tratamentos. A contagem do número de insetos por planta foi realizada com 5, 10, 24, 48 e 120 horas após a aplicação utilizando-se, sob as plantas, um lençol plástico preto, medindo 2 x $2 \mathrm{~m}$. Os mais eficientes foram fenvalerato $30 \mathrm{CE}(83,72 \%$ e deltametrina 2,5 CE $(79,42 \%)$, os quais diferiram estatisticamente dos demais, porém não diferiram entre si. 